\title{
Thermolysis of Organofluoroborate Ionic Liquids to NHC-Organofluoroborates
}

Coby J. Clarke, ${ }^{1,2 *}$ Nathan D. Richardson, ${ }^{2}$ Anton, E. J. Firth, ${ }^{1}$ Jason P. Hallett ${ }^{l}$ and Peter Licence $^{2}$

*Correspondence:

coby.clarke@nottingham.ac.uk

Affiliations:

1 Department of Chemical Engineering, Imperial College London, South Kensington Campus, Imperial College Road, Kensington, London, SW7 2AZ, UK

2 Glaxo Smith Kline Carbon Neutral Laboratory for Sustainable Chemistry, University of Nottingham, Jubilee Campus, 6 Triumph Rd, Lenton, Nottingham NG7 2GA, UK

KEYWORDS: Ionic liquids, thermolysis, organofluoroborates, direct insertion mass spectrometry, tetrafluoroborates 


\begin{abstract}
A range of dialkylimidazolium organotrifluoroborate ionic liquids were prepared by anion metathesis from newly available potassium organotrifluoroborate salts, and their physical properties were characterized by TGA, DSC, and STA. Thermal decomposition was subsequently investigated under vacuum with direct insertion mass spectrometry to determine whether thermolysis was a viable route to prepare N-heterocyclic carbene organofluoroborates, which are an important class of emerging compounds. Several key targets were identified, which highlighted the potential to short-cut complex synthetic methodologies otherwise required to access such molecules.
\end{abstract}




\section{Introduction}

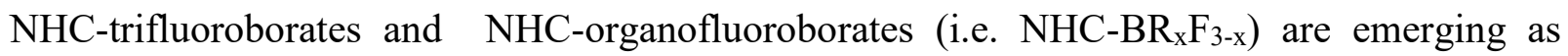
valuable new molecules with unique reactivity, which are impacting areas such as synthetic organic chemistry, ${ }^{1,2}$ polymer chemistry, ${ }^{3}$ photoelectrochemistry, ${ }^{4}$ and PET imaging. ${ }^{5,6}$ In many cases, $\mathrm{NHC}-\mathrm{BR}_{\mathrm{x}} \mathrm{F}_{3-\mathrm{x}}$ compounds are replacing expensive metal catalysts and their applications are rapidly growing as their chemistry is unraveled. $\mathrm{NHC}-\mathrm{BR}_{\mathrm{x}} \mathrm{F}_{3-\mathrm{x}}$ compounds can be prepared from free $\mathrm{NHCs}_{\text {and }} \mathrm{BR}_{\mathrm{x}} \mathrm{F}_{3-\mathrm{x}}$; however this method requires strong bases which limits the flexibility of

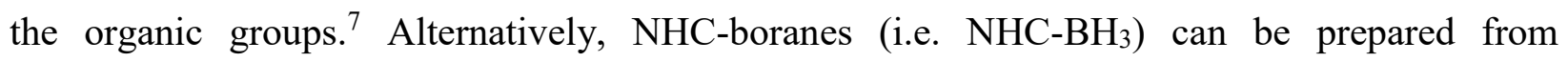
imidazolium salts and sodium borohydride, ${ }^{8}$ and aryl-substituted NHC-boranes can then be prepared by hydroboration to arynes, ${ }^{9}$ followed by fluorination to afford $\mathrm{NHC}-\mathrm{BRF}_{2}{ }^{1}$ Unfortunately, each step requires organic solvents, harmful reagents, industrially challenging work up procedures, and have low atom economies

Most ionic liquids decompose via mechanisms such as retro- $\mathrm{S}_{\mathrm{N}} 2$ that yield starting materials or small molecules; ${ }^{10,11}$ however, thermolysis of imidazolium tetrafluoroborate (i.e. $\left.\left[\mathrm{C}_{n} \mathrm{C}_{1} \operatorname{Im}\right]\left[\mathrm{BF}_{4}\right]\right)$ ionic liquids (ILs) under high vacuum has been shown to be a viable route to prepare high-value NHC-trifluoroborates (i.e. $\mathrm{NHC}^{-\mathrm{BF}_{3}}$; SI, Scheme S1a). ${ }^{12}$ Here, we prepare and characterize new organofluoroborate ILs (i.e. $\left[\mathrm{C}_{4} \mathrm{C}_{1} \mathrm{Im}\right]\left[\mathrm{BR}_{\mathrm{x}} \mathrm{F}_{4-\mathrm{x}}\right]$ ) from newly available potassium organotrifluoroborate (i.e. $\mathrm{K}\left[\mathrm{R}-\mathrm{BF}_{3}\right]$ ) salts, ${ }^{13}$ and investigate their thermal behavior to understand whether they are suitable precursors for $\mathrm{NHC}_{-} \mathrm{BR}_{\mathrm{x}} \mathrm{F}_{3-\mathrm{x}}$ synthesis (Scheme S1b). Electron ionization mass spectrometry (EI-MS) has previously been used to identify NHC-adducts from known fragmentation patterns $\left(\mathrm{NHC}_{-3 F} \rightarrow \mathrm{NHC}_{3}-\mathrm{BF}_{2}{ }^{+\bullet} ; \mathrm{SI}\right.$, Scheme S1c) ${ }^{14}$ We exploit this knowledge to identify vapour phase thermal decomposition $\left(T_{d}\right)$ products from in-situ measurements with direct insertion mass spectrometry (DIMS). Heating ILs under reduced 
pressure often leads to competitive vaporization and $T_{d}$, which can be monitored by MS signals of vaporized IL (i.e. neutral ion pairs, NIPs),${ }^{15} \mathrm{NHC}$ adducts,,${ }^{14}$ or $\mathrm{S}_{\mathrm{N}} 2 T_{d}$ products. ${ }^{16}$ Crucially, thermolysis can be a valuable synthetic short-cut to avoid numerous solvents and auxiliaries, but thermal behavior must first be fully understood to realize such possibilities.

\section{Experimental}

$\left[\mathrm{C}_{4} \mathrm{C}_{1} \operatorname{Im}\right]\left[\mathrm{BR}_{\mathrm{x}} \mathrm{F}_{4-\mathrm{x}}\right] \mathrm{ILs}$ were synthesized by anion metathesis from $\left[\mathrm{C}_{4} \mathrm{C}_{1} \mathrm{Im}\right] \mathrm{Cl}$ in anhydrous DCM and characterized by NMR and HRMS. Relevant structures are shown in Figure 1 and Figure S1, and ILs are labelled IL1-11 according to Table 1. All ILs were analyzed by differential scanning calorimetry (DSC), thermogravimetric analysis (TGA), and simultaneous thermal analysis (STA), and finally by DIMS (full details in the supporting information).

\section{Results and Discussion}

All ILs were first characterized by DSC, TGA, and STA to compare the effects of organo-groups on thermal properties (Table 1). Six of the seven $\left[\mathrm{C}_{4} \mathrm{C}_{1} \mathrm{Im}\right]\left[\mathrm{BRF}_{3}\right]$ ILs were found to be room temperature ILs by DSC. Butyl- (IL3) and cyclohexyl- (IL8) groups had the largest impacts by introducing melting points $\left(T_{m}\right)$ that were $109.4{ }^{\circ} \mathrm{C}$ and $156.1{ }^{\circ} \mathrm{C}$ higher than the $T_{g}$ of $\left[\mathrm{C}_{4} \mathrm{C}_{1} \operatorname{Im}\right]\left[\mathrm{BF}_{4}\right]$, respectively. Conversely, the single aromatic groups of IL5-7 only raised the glass transition $\left(T_{g}\right)$ temperature by $22-28{ }^{\circ} \mathrm{C}$, while the four phenyl groups of IL9 introduced a $T_{m} 223.3$ ${ }^{\circ} \mathrm{C}$ above the $T_{g}$ of $\left[\mathrm{C}_{4} \mathrm{C}_{1} \mathrm{Im}\right]\left[\mathrm{BF}_{4}\right]$. Except for $\left[\mathrm{B}\left(\mathrm{C}_{6} \mathrm{H}_{5}\right)\right]^{-}$(i.e. IL-9), the organic groups compromised IL thermal stability by introducing exothermic $T_{d}$ steps at significantly lower temperatures than those associated with endothermic $\left[\mathrm{BF}_{4}\right]^{-} T_{d}$ (SI, Figure S26-36). Hence, $\left[\mathrm{C}_{4} \mathrm{C}_{1} \operatorname{Im}\right]\left[\mathrm{BRF}_{3}\right]$ TGA $T_{d}$ parameters were lower than those of $\left[\mathrm{C}_{4} \mathrm{C}_{1} \operatorname{Im}\right]\left[\mathrm{BF}_{4}\right]$, and the lowest 
values were obtained for IL6 while the highest was obtained for IL5, which highlighted that strong electron withdrawing groups were detrimental to $\left[\mathrm{RBF}_{3}\right]^{-}$thermal stability. A dicationic analogue (IL11) had the highest stability as expected, ${ }^{17}$ and a hydroxyl-functionalized IL cation (IL10) also appeared to have higher stability than all $\left[\mathrm{C}_{4} \mathrm{C}_{1} \mathrm{Im}\right]\left[\mathrm{BRF}_{3}\right]$ ILs. Isothermal TGA of allylfunctionalized $\left[\mathrm{C}_{4} \mathrm{C}_{1} \mathrm{Im}\right]\left[\left(\mathrm{C}_{3} \mathrm{H}_{5}\right) \mathrm{BF}_{3}\right]$ highlighted that organo groups also had an impact on longterm thermal stability (SI, Figure S37-39). For example, the temperatures at which $1 \%$ mass loss occurred over $10 \mathrm{hr}\left(\mathrm{T}_{0.01 / 10}\right)$ was $167^{\circ} \mathrm{C}$, which was $49{ }^{\circ} \mathrm{C}$ lower than the literature value for $\left[\mathrm{C}_{4} \mathrm{C}_{1} \mathrm{Im}\right]\left[\mathrm{BF}_{4}\right] .{ }^{18}$ However, the activation energy $\left(E_{a}\right)$ of $T_{d}$ only decreased by $9.5 \mathrm{~kJ} \mathrm{~mol}^{-1}$ from $108.4 \mathrm{~kJ} \mathrm{~mol}^{-1}$ to $98.9 \mathrm{~kJ} \mathrm{~mol}^{-1}$. In comparison, hydroxyl-functionalised IL10 had a relatively similar $\mathrm{T}_{0.01 / 10}$ value of $166^{\circ} \mathrm{C}$ (SI, Figure S40-42), but a significantly lower $\mathrm{E}_{\mathrm{a}}$ of $85.1 \mathrm{~kJ} \mathrm{~mol}^{-1}$, which was below the values of low thermal stability ILs. ${ }^{19}$ Therefore, functional groups compromised atmospheric pressure thermal stability, regardless of whether they were situation on the IL anion (i.e. $\left.\left[\left(\mathrm{C}_{3} \mathrm{H}_{5}\right) \mathrm{BF}_{3}\right]^{-}\right)$or cation (i.e. $\left.\left[\left(\mathrm{C}_{2} \mathrm{OH}\right) \mathrm{C}_{1} \mathrm{Im}\right]^{+}\right)$. 
Table 1. Atmospheric pressure phase transitions $\left(T_{m}, T_{g}, T_{c c}\right)$ measured by $D S C$, thermal decompositions ( $\left.\mathrm{T}_{\text {onset, }} \mathrm{T}_{1 \%}\right)$ measured by TGA, number of steps in the TGA, STA signal for the initial thermal decomposition step.

\begin{tabular}{|c|c|c|c|c|c|c|c|c|c|c|}
\hline \multirow[b]{2}{*}{ IL } & \multirow[b]{2}{*}{ Cation } & \multirow[b]{2}{*}{ Anion } & \multirow[b]{2}{*}{$T_{m}$} & \multicolumn{5}{|c|}{ Temperature $/{ }^{\circ} \mathrm{C}$} & \multirow[b]{2}{*}{ No. Steps } & \multirow[b]{2}{*}{ Initial Step } \\
\hline & & & & $T_{g}$ & $T_{c c}$ & $T_{s s}$ & $T_{\text {onset }}$ & $T_{1 \%}$ & & \\
\hline 1 & {$\left[\mathrm{C}_{4} \mathrm{C}_{1} \mathrm{Im}\right]^{+}$} & {$\left[\mathrm{BF}_{4}\right]^{-}$} & & $-94.2^{\mathrm{a}}$ & & & 429.7 & 367.5 & 1 & endothermic \\
\hline 2 & {$\left[\mathrm{C}_{4} \mathrm{C}_{1} \mathrm{Im}\right]^{+}$} & {$\left[\left(\mathrm{CH}_{3}\right) \mathrm{BF}_{3}\right]^{-}$} & & -95.3 & & & 299.9 & 195.0 & 2 & exothermic \\
\hline 3 & {$\left[\mathrm{C}_{4} \mathrm{C}_{1} \mathrm{Im}\right]^{+}$} & {$\left[\left(\mathrm{C}_{4} \mathrm{H}_{9}\right) \mathrm{BF}_{3}\right]^{-}$} & 15.2 & -79.1 & -37.9 & & 276.9 & 260.4 & $2-3$ & exothermic \\
\hline 4 & {$\left[\mathrm{C}_{4} \mathrm{C}_{1} \mathrm{Im}\right]^{+}$} & {$\left[\left(\mathrm{C}_{3} \mathrm{H}_{5}\right) \mathrm{BF}_{3}\right]^{-}$} & & -92.2 & & & 242.7 & 205.3 & 2 & exothermic \\
\hline 5 & {$\left[\mathrm{C}_{4} \mathrm{C}_{1} \mathrm{Im}\right]^{+}$} & {$\left[\left(\mathrm{C}_{6} \mathrm{H}_{5}\right) \mathrm{BF}_{3}\right]^{-}$} & & -71.6 & & & 309.4 & 287.2 & 2 & exothermic \\
\hline 6 & {$\left[\mathrm{C}_{4} \mathrm{C}_{1} \mathrm{Im}\right]^{+}$} & {$\left[\left(\mathrm{C}_{6} \mathrm{H}_{3} \mathrm{~F}_{2}\right) \mathrm{BF}_{3}\right]^{-}$} & & -69.9 & & & 233.8 & 198.7 & $2-3$ & exothermic \\
\hline 7 & {$\left[\mathrm{C}_{4} \mathrm{C}_{1} \mathrm{Im}\right]^{+}$} & {$\left[\left(\mathrm{C}_{7} \mathrm{H}_{6} \mathrm{OH}\right) \mathrm{BF}_{3}\right]^{-}$} & & -66.6 & & & 270.4 & 178.6 & $2-3$ & exothermic \\
\hline 8 & {$\left[\mathrm{C}_{4} \mathrm{C}_{1} \mathrm{Im}\right]^{+}$} & {$\left[\left(\mathrm{C}_{6} \mathrm{H}_{11}\right) \mathrm{BF}_{3}\right]^{-}$} & $61.9^{\mathrm{b}}$ & $-53.5^{b}$ & $-13.1^{b}$ & $26.3^{\mathrm{b}}$ & 316.1 & 267.9 & 2 & exothermic \\
\hline 9 & {$\left[\mathrm{C}_{4} \mathrm{C}_{1} \mathrm{Im}\right]^{+}$} & {$\left[\mathrm{B}\left(\mathrm{C}_{6} \mathrm{H}_{5}\right)_{4}\right]^{-}$} & $129.1^{\mathrm{c}}$ & $-88.1^{\mathrm{d}}$ & & & 349.6 & 324.1 & 1 & endothermic \\
\hline 10 & {$\left[\left(\mathrm{C}_{2} \mathrm{OH}\right) \mathrm{C}_{1} \mathrm{Im}\right]^{+}$} & {$\left[\mathrm{BF}_{4}\right]^{-}$} & & -87.8 & & & 360.5 & 265.0 & 1 & endothermic \\
\hline 11 & {$\left[\mathrm{C}_{12}\left(\mathrm{C}_{1} \mathrm{Im}\right)_{2}\right]^{2+}$} & $2\left[\mathrm{BF}_{4}\right]^{-}$ & 2.4 & -49.7 & -23.6 & & 440.2 & 288.3 & 1 & endothermic \\
\hline
\end{tabular}

${ }^{\mathrm{a}}$ Literature value of $-85.0^{\circ} \mathrm{C}^{20}$

${ }^{b}$ Resolved at $20^{\circ} \mathrm{C} / \mathrm{min}$

${ }^{\mathrm{c}}$ Amorphous m.p. of $129.1{ }^{\circ} \mathrm{C}$ from STA

${ }^{\mathrm{d}} \mathrm{T}_{\mathrm{g}}$ of the supercooled liquid; solid at R.T.

All ILs were next investigated by DIMS to probe their thermal behavior under vacuum (Figure

1a-k; SI, Figure S43-53). DIMS of the control IL1 (Figure 1a) showed that both vaporization and $T_{d}$ occurred simultaneously as $\mathrm{m} / \mathrm{z}$ signals for NIPs (i.e. $\mathrm{m} / \mathrm{z} 139$ for $\left.\left[\mathrm{C}_{4} \mathrm{C}_{1} \operatorname{Im}\right]^{+}\right)^{21,22}$ and $\left[\mathrm{C}_{4} \mathrm{C}_{1} \operatorname{Im}\right]$ $\mathrm{BF}_{3}$ zwitterions (i.e. m/z 187 for $\left.\left[\mathrm{C}_{4} \mathrm{C}_{1} \mathrm{Im}\right]-\mathrm{BF}_{2}{ }^{+\bullet}\right)^{14}$ were observed. For dicationic IL11, no NHC$\mathrm{BF}_{3}$ adducts were observed and all $T_{d}$ species were identified as alkyl-chain fragmentation 
products (Figure 1k). ${ }^{22}$ This was supported by $\mathrm{CH}_{2}$-loss peaks observed in the MS and the presence of other imidazolium fragmentation products (i.e. $\mathrm{m} / \mathrm{z} 82,137$ ).

All $\left[\mathrm{C}_{4} \mathrm{C}_{1} \mathrm{Im}\right]\left[\mathrm{BR}_{\mathrm{x}} \mathrm{F}_{4-\mathrm{x}}\right]$ ILs vaporized or decomposed (Figure $\mathbf{1 b}-\mathbf{k}$ ) far below IL1 which had an onset of $\approx 300{ }^{\circ} \mathrm{C}$. For IL2 (Figure $\mathbf{1 b}$ ), the $\mathrm{m} / \mathrm{z}$ signal of $\left[\mathrm{C}_{4} \mathrm{C}_{1} \mathrm{Im}\right]-\mathrm{BF}_{2}{ }^{+\bullet}$ appeared around 125 ${ }^{\circ} \mathrm{C}$ and peaked at $250{ }^{\circ} \mathrm{C}$ before decreasing. This was $\approx 175{ }^{\circ} \mathrm{C}$ below IL1 which suggested that $\left[\mathrm{C}_{4} \mathrm{C}_{1} \mathrm{Im}\right]-\mathrm{BF}_{3}$ was formed at low temperatures when fluorine was substituted for a methyl group. An additional fragmentation product $\left[\mathrm{C}_{4} \mathrm{C}_{1} \mathrm{Im}\right]-\mathrm{B}\left(\mathrm{CH}_{3}\right) \mathrm{F}^{+\bullet}$ was also observed, albeit with very low intensity, which suggested that $\left[\mathrm{C}_{4} \mathrm{C}_{1} \mathrm{Im}\right]-\mathrm{B}\left(\mathrm{CH}_{3}\right) \mathrm{F}_{2}$ had formed through $T_{d}$ of the IL. It was unclear whether $\left[\mathrm{C}_{4} \mathrm{C}_{1} \mathrm{Im}\right]-\mathrm{BF}_{3}$ was the major $\mathrm{T}_{\mathrm{d}}$ product or whether $\left[\mathrm{C}_{4} \mathrm{C}_{1} \mathrm{Im}\right]-\mathrm{BF}_{2}{ }^{+\bullet}$ was the most stable fragmentation product and $\left[\mathrm{C}_{4} \mathrm{C}_{1} \mathrm{Im}\right]-\mathrm{B}\left(\mathrm{CH}_{3}\right) \mathrm{F}_{2}$ had formed but was not readily identified. Low $\mathrm{MW}\left(\mathrm{CH}_{3}\right) \mathrm{BF}_{2}^{+\bullet}$ was also identified in the IL vapour, which could have originated from either $\left[\mathrm{C}_{4} \mathrm{C}_{1} \mathrm{Im}\right]-\mathrm{B}\left(\mathrm{CH}_{3}\right) \mathrm{F}_{2}$ fragmentation or NIPs (i.e. $\left.\left[\left(\mathrm{CH}_{3}\right) \mathrm{BF}_{3}\right]^{-}\right)$. 
(a)

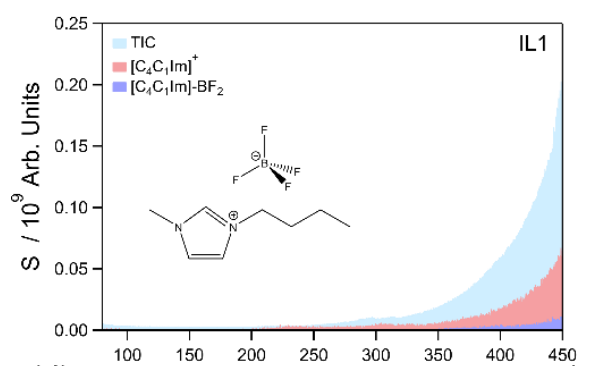

(d)

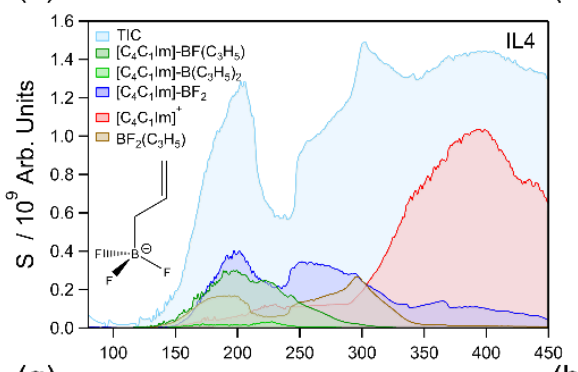

(g)

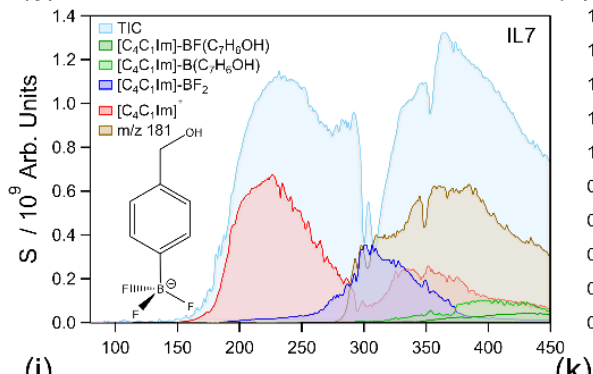

(j)

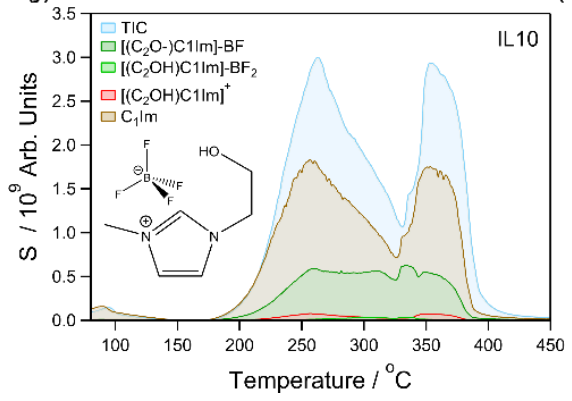

(b)

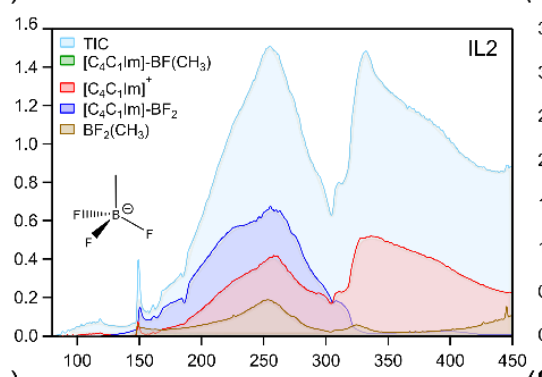

(e)

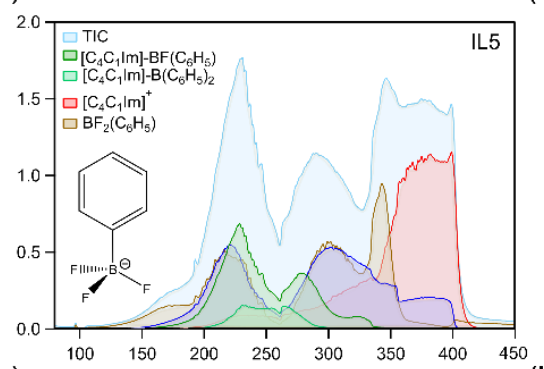

(h)

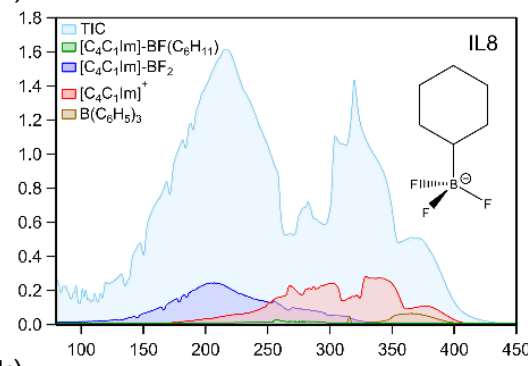

(k)

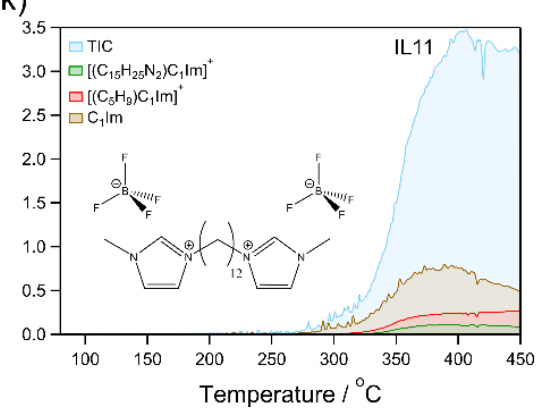

(c)

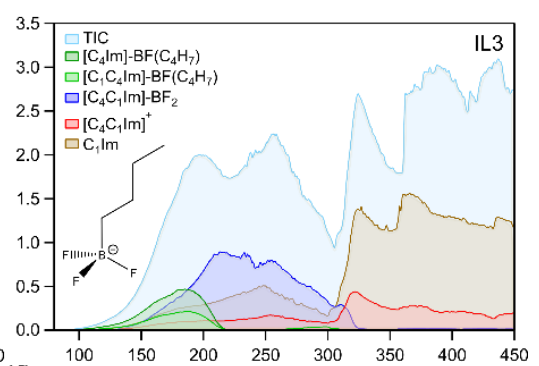

(f)

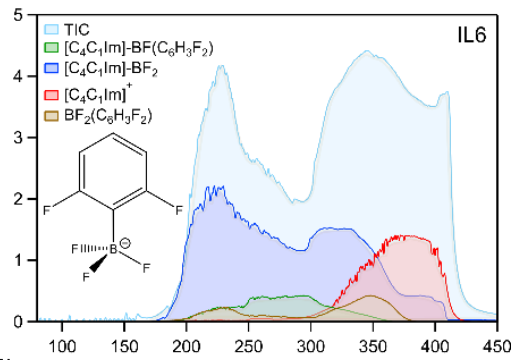

(i)

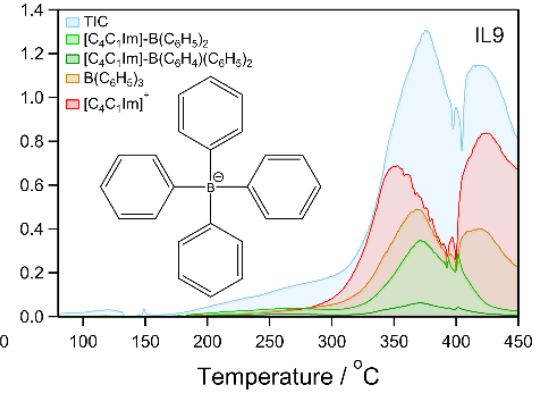

Figure 1. Total ion currents (blue) and selected $m / z$ profiles for DIMS of $\left[\mathrm{C}_{4} \mathrm{C}_{1} \mathrm{Im}\right]\left[\mathrm{BF}_{4}\right](\mathrm{a})$, $\left[\mathrm{C}_{4} \mathrm{C}_{1} \operatorname{Im}\right]\left[\mathrm{BR}_{\mathrm{x}} \mathrm{F}_{4-\mathrm{x}}\right]$ where $\mathrm{x}=1$ or $4(\mathrm{~b}-\mathrm{i})$ with anion structures only, $\left[\left(\mathrm{C}_{2} \mathrm{OH}\right) \mathrm{C}_{1} \operatorname{Im}\right]\left[\mathrm{BF}_{4}\right](\mathrm{j})$, and $\left[\mathrm{C}_{12}\left(\mathrm{C}_{1} \mathrm{Im}\right)_{2}\right]\left[\mathrm{BF}_{4}\right]_{2}$ at $10^{\circ} \mathrm{C} / \mathrm{min}$.

The first two $\mathrm{m} / \mathrm{z}$ signals from IL3 appeared between $100{ }^{\circ} \mathrm{C}$ and $225^{\circ} \mathrm{C}$ and were a result of $T_{d}$ of the IL to $\left[\mathrm{C}_{4} \mathrm{C}_{1} \mathrm{Im}\right]-\mathrm{BF}_{2}\left(\mathrm{C}_{4} \mathrm{H}_{9}\right)$ (Figure $\left.1 \mathrm{c}\right) . \mathrm{A}$ broad peak for $\left[\mathrm{C}_{4} \mathrm{C}_{1} \mathrm{Im}\right]-\mathrm{BF}_{2}{ }^{+\bullet}$ was observed from 
$118^{\circ} \mathrm{C}$ to $325^{\circ} \mathrm{C}$, and this was subsequently followed by NIP signals from $325^{\circ} \mathrm{C}$ to $450{ }^{\circ} \mathrm{C}$. The high intensity of $\mathrm{C}_{1} \mathrm{Im}$ relative to $\left[\mathrm{C}_{4} \mathrm{C}_{1} \mathrm{Im}\right]^{+}$suggested that IL $T_{d}$ was occurring via an $\mathrm{S}_{\mathrm{N}} 2$ mechanism. ${ }^{16}$ DIMS of IL4 (Figure 1d) supported the results from IL3 by further confirming that the $\mathrm{NHC}-\mathrm{BRF}_{2}$ product was preferentially formed at low temperature between $125^{\circ} \mathrm{C}$ to $300^{\circ} \mathrm{C}$. Interestingly, a disubstituted $\left[\mathrm{C}_{4} \mathrm{C}_{1} \operatorname{Im}\right]-\mathrm{BF}\left(\mathrm{C}_{3} \mathrm{H}_{5}\right)_{2}{ }^{+\bullet}$ radical cation was also identified, which highlighted that additional $T_{d}$ processes were occurring before formation of volatile $\mathrm{NHC}-\mathrm{BRF}_{2}$. The high intensity of $\left[\mathrm{C}_{4} \mathrm{C}_{1} \mathrm{Im}\right]^{+}$at $>300{ }^{\circ} \mathrm{C}$ showed that the vapour was primarily composed of intact NIPs.

IL5 showed similar trends (Figure 1e) to those observed for IL4. Between $150-250{ }^{\circ} \mathrm{C}, T_{d}$ of $\left[\mathrm{C}_{4} \mathrm{C}_{1} \operatorname{Im}\right]\left[\left(\mathrm{C}_{6} \mathrm{H}_{5}\right) \mathrm{BF}_{3}\right]$ gave a mixture of $\left[\mathrm{C}_{4} \mathrm{C}_{1} \operatorname{Im}\right]-\mathrm{BF}\left(\mathrm{C}_{6} \mathrm{H}_{5}\right)^{+\bullet}, \quad\left[\mathrm{C}_{4} \mathrm{C}_{1} \operatorname{Im}\right]-\mathrm{B}\left(\mathrm{C}_{6} \mathrm{H}_{5}\right)_{2}{ }^{+\bullet}$, and $\left[\mathrm{C}_{4} \mathrm{C}_{1} \mathrm{Im}\right]-\mathrm{BF}_{2}{ }^{+\bullet}$. As the temperature increased to $>300{ }^{\circ} \mathrm{C},\left[\mathrm{C}_{4} \mathrm{C}_{1} \mathrm{Im}\right]-\mathrm{BF}_{2}{ }^{+\bullet}$ was still present, but $\left[\mathrm{C}_{4} \mathrm{C}_{1} \mathrm{Im}\right]^{+} \mathrm{NIPs}$ dominated the $\mathrm{MS} ; \mathrm{BF}_{2}\left(\mathrm{C}_{6} \mathrm{H}_{5}\right)^{+\bullet}$ was present throughout heating. For IL6, the fluorinated phenyl-ring resulted in larger amounts of $\left[\mathrm{C}_{4} \mathrm{C}_{1} \mathrm{Im}\right]-\mathrm{BF}_{2}{ }^{+\bullet}$ at low temperature (Figure 1f) compared to non-fluorinated analogue IL5. Although a relatively small amount of $\left[\mathrm{C}_{4} \mathrm{C}_{1} \operatorname{Im}\right]$ $\mathrm{BF}\left(\mathrm{C}_{6} \mathrm{H}_{3} \mathrm{~F}_{2}\right)^{+\bullet}$ was present in the vapour from $200{ }^{\circ} \mathrm{C}$ to $350{ }^{\circ} \mathrm{C}$, no disubstituted $\left[\mathrm{C}_{4} \mathrm{C}_{1} \mathrm{Im}\right]-$ $\mathrm{B}\left(\mathrm{C}_{6} \mathrm{H}_{3} \mathrm{~F}_{2}\right)_{2}{ }^{+\bullet}$ was observed. Again, it was unclear whether the increased electron withdrawing properties of the organo-group were influencing $T_{d}$ processes, or El fragmentation processes.

Unlike all other ILs, IL7 showed $\left[\mathrm{C}_{4} \mathrm{C}_{1} \mathrm{Im}\right]^{+}$was present at relatively low temperature from 150 ${ }^{\circ} \mathrm{C}$ (Figure 1g). The $T_{d}$ product $\left[\mathrm{C}_{4} \mathrm{C}_{1} \mathrm{Im}\right]-\mathrm{BF}_{3}$ was only produced at higher temperatures which peaked at $300{ }^{\circ} \mathrm{C}$, then steadily decreased. Both $\left[\mathrm{C}_{4} \mathrm{C}_{1} \mathrm{Im}\right]-\mathrm{B}\left(\mathrm{C}_{7} \mathrm{H}_{6} \mathrm{OH}\right)^{+\bullet}$ and $\left[\mathrm{C}_{4} \mathrm{C}_{1} \mathrm{Im}\right]-\mathrm{BF}\left(\mathrm{C}_{7} \mathrm{H}_{6} \mathrm{OH}\right)$ 
+• were observed which indicated the presence of $\left[\mathrm{C}_{4} \mathrm{C}_{1} \mathrm{Im}\right]-\mathrm{BF}_{2}\left(\mathrm{C}_{7} \mathrm{H}_{6} \mathrm{OH}\right)$, albeit at very high temperatures $\left(325^{\circ} \mathrm{C}\right.$ to $\left.450{ }^{\circ} \mathrm{C}\right)$. An unidentified decomposition product was also observed (m/z 181) from $275{ }^{\circ} \mathrm{C}$ to $450{ }^{\circ} \mathrm{C}$, which further highlighted the complexity of $T_{d}$ when reactive functional groups were present. IL8 gave a low intensity signal for $\left[\mathrm{C}_{4} \mathrm{C}_{1} \mathrm{Im}\right]-\mathrm{BF}\left(\mathrm{C}_{6} \mathrm{H}_{11}\right)^{+\bullet}($ Figure 1h) but had the lowest onset temperature for $\left[\mathrm{C}_{4} \mathrm{C}_{1} \mathrm{Im}\right]-\mathrm{BF}_{3}$ production, which was present in the $80{ }^{\circ} \mathrm{C}$ drying step before heating was started. The dehydrogenation product $\mathrm{B}\left(\mathrm{C}_{6} \mathrm{H}_{5}\right)_{3}{ }^{+\bullet}$ was observed at high temperature $\left(325^{\circ} \mathrm{C}\right.$ to $\left.400^{\circ} \mathrm{C}\right)$. The tetraphenylborate anion in IL9 gave a reasonably high vacuum $T_{d}$ (Figure 1i), albeit with low levels of signal $<300{ }^{\circ} \mathrm{C}$. The presence of $\left[\mathrm{C}_{4} \mathrm{C}_{1} \mathrm{Im}\right]-\mathrm{B}\left(\mathrm{C}_{6} \mathrm{H}_{5}\right)_{3}$ fragmentation products showed that NHC-BR 3 adducts could form through $T_{d}$. The fragmentation product $\mathrm{B}\left(\mathrm{C}_{6} \mathrm{H}_{5}\right)_{3}{ }^{+\bullet}$ was observed throughout and could have originated from NIPs or $\left[\mathrm{C}_{4} \mathrm{C}_{1} \mathrm{Im}\right]-\mathrm{B}\left(\mathrm{C}_{6} \mathrm{H}_{5}\right)_{3}$.

The hydroxyl-functionalized cation of IL10 produced only two major signals (Figure 1j), which suggested that the IL had relatively simple $T_{d}$ behavior. The vapour was found to consist of $\mathrm{C}_{1} \mathrm{Im}$, an expected decomposition product from loss of $-\mathrm{C}_{2} \mathrm{OH}$, and $\left[\left(\mathrm{C}_{2} \mathrm{O}-\right) \mathrm{C}_{1} \mathrm{Im}\right]-\mathrm{BF}^{+\bullet}($ Figure $2 \mathbf{f})$ which was identified following EI fragmentation rules. Low levels of NIPs were present in the vapour and a low intensity signal for $\left[\left(\mathrm{C}_{2} \mathrm{OH}\right) \mathrm{C}_{1} \mathrm{Im}\right]-\mathrm{BF}_{2}$ was observed. In total six key $N H C-\mathrm{BR}_{\mathrm{x}} \mathrm{F}_{3-\mathrm{x}}$ targets were identified as potential high-value products (Figure 2), including the cyclic compound identified from IL10. We are currently undertaking subsequent steps involving the scale-up and isolation of such compounds in a bespoke set-up to optimize production. 

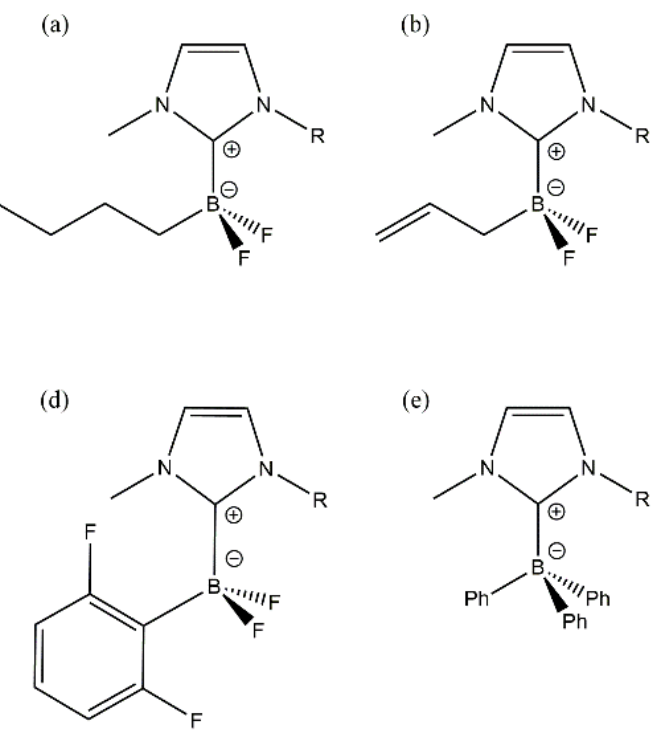

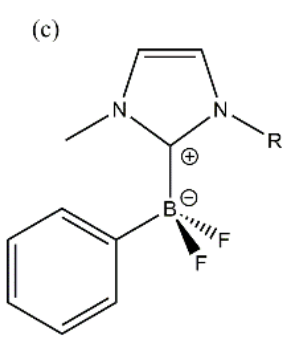

(e)

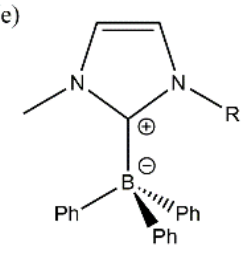

(f)

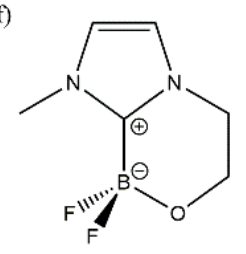

Figure 2. NHC-BR $\mathrm{F}_{3-\mathrm{x}}$ products identified as key targets from DIMS experiments.

\section{Conclusions}

A range of $\left[\mathrm{C}_{4} \mathrm{C}_{1} \mathrm{Im}\right]\left[\mathrm{BR}_{\mathrm{x}} \mathrm{F}_{4-\mathrm{x}}\right]$ ILs were prepared and thermally characterized. In all cases, organo-groups compromised thermal stabilities by introducing anion-led $T_{d}$ steps far below $\left[\mathrm{BF}_{4}\right]^{-}$ $T_{d}$. DIMS showed that vacuum thermolysis was a viable route to prepare high value $\mathrm{NHC}-\mathrm{BR}_{\mathrm{x}} \mathrm{F}_{3-\mathrm{x}}$ compounds if the organic groups were thermally robust. In vacuum, all $\left[\mathrm{C}_{4} \mathrm{C}_{1} \mathrm{Im}\right]\left[\mathrm{BR}_{\mathrm{x}} \mathrm{F}_{4-\mathrm{x}}\right]$ ILs decomposed at lower temperatures than $\left[\mathrm{C}_{4} \mathrm{C}_{1} \operatorname{Im}\right]\left[\mathrm{BF}_{4}\right]$, and mixtures of NIPs, NHC-BR $\mathrm{F}_{3-\mathrm{x}}$, and $\mathrm{S}_{\mathrm{N}} 2 T_{d}$ products were identified. Six $\left[\mathrm{C}_{4} \mathrm{C}_{1} \mathrm{Im}\right]\left[\mathrm{BR}_{\mathrm{x}} \mathrm{F}_{4-\mathrm{x}}\right]$ ILs were also identified as key precursors to novel NHC-BR ${ }_{x} \mathrm{~F}_{-\mathrm{x}}$ compounds because $\mathrm{NHC}-\mathrm{BR}_{\mathrm{x}} \mathrm{F}_{3-\mathrm{x}}$ was either produced at low temperatures or in high quantity. Although some ILs did not produce $\mathrm{NHC}-\mathrm{BR}_{\mathrm{x}} \mathrm{F}_{3-\mathrm{x}}$, they may serve as ideal precursors to $\mathrm{NHC}_{-} \mathrm{BF}_{3}$ compounds because of their potential to liberate $\mathrm{NHC}-\mathrm{BF}_{3}$ at low temperatures - likely because B-C bonds are significantly weaker than B-F bonds. ${ }^{3}$ Dicationic $\left[\mathrm{C}_{12}\left(\mathrm{C}_{1} \mathrm{Im}\right)_{2}\right][\mathrm{BF}]_{2}$ decomposed by chain fragmentation, but hydroxyl-functionalised $\left[\left(\mathrm{C}_{2} \mathrm{OH}\right) \mathrm{C}_{1} \mathrm{Im}\right]\left[\mathrm{BF}_{4}\right]$ produced significant quantities of a cyclic $\mathrm{NHC}-\mathrm{BR}_{\mathrm{x}} \mathrm{F} 3-\mathrm{x}$ where the organic group was from the imidazolium alkyl-chain. By identifying key targets, we present the first steps 
in realizing thermolysis as a viable short-cut to high value compounds that may bypass significant quantities of solvents, auxiliaries, and work-up procedures.

\section{ASSOCIATED CONTENT}

\section{Supporting Information.}

The following files are available free of charge.

Experimental data including synthesis and characterization of ionic liquids, STA, TGA, DIMS, and TGA-MS data (PDF).

\section{AUTHOR INFORMATION}

\section{Corresponding Author}

* coby.clarke@nottingham.ac.uk

\section{Author Contributions}

Experiments were performed by CJC, NDR, and AEJF. Data was interpreted by CJC and NDR and the manuscript was written by CJC. All authors have given approval to the final version of the manuscript.

\section{Funding Sources}

JPH would like to acknowledge EPSRC EP/K038648/1, and PL would like to acknowledge EP/K005138/1 for support.

\section{Notes}

The authors declare no competing financial interest. 


\title{
ACKNOWLEDGMENT
}

CJC would like to thank Dr Kevin Lovelock for $\left[\left(\mathrm{C}_{2} \mathrm{OH}\right) \mathrm{C}_{1} \mathrm{Im}\right]\left[\mathrm{BF}_{4}\right]$.

\begin{abstract}
ABBREVIATIONS
ILs, ionic liquids; PET, positron emission tomography; EI-MS, electron ionization mass spectrometry; $\mathrm{T}_{\mathrm{d}}$, thermal decomposition; DIMS, direct insertion mass spectrometry; NIPs, neutral ion pairs; DSC, differential scanning calorimetry; TGA, thermogravimetric analysis; STA, simultaneous thermal analysis; $\mathrm{T}_{\mathrm{m}}$, melting temperature; $\mathrm{T}_{\mathrm{g}}$, glass transition temperature; $E_{a}$, activation energy; $\mathrm{T}_{0.01 / 10}$, temperature at which $1 \%$ mass is lost over 10 hours; $\mathrm{T}_{\mathrm{cc}}$, cold crystallization temperature; $\mathrm{T}_{\mathrm{ss}}$, solid-solid transition temperature; $\mathrm{T}_{\text {onset, }}$, onset of thermal decomposition; $\mathrm{T}_{1 \%}, 1 \%$ mass loss temperature;
\end{abstract}

\section{REFERENCES}

(1) Nerkar, S.; Curran, D. P. Synthesis and Suzuki Reactions of N-Heterocyclic Carbene Difluoro(Aryl)-Boranes. Org. Lett. 2015, $17 \quad$ (14), 3394-3397. https://doi.org/10.1021/acs.orglett.5b01101.

(2) Shimoi, M.; Watanabe, T.; Maeda, K.; Curran, D. P.; Taniguchi, T. Radical TransHydroboration of Alkynes with N-Heterocyclic Carbene Boranes. Angew. Chem. Int. Ed. 2018, 57 (30), 9485-9490. https://doi.org/10.1002/anie.201804515.

(3) Subervie, D.; Graff, B.; Nerkar, S.; Curran, D. P.; Lalevée, J.; Lacôte, E. Difluorination at Boron Leads to the First Electrophilic Ligated Boryl Radical (NHC-BF2.). Angew. Chem. 
2018, 130 (32), 10408-10413. https://doi.org/10.1002/ange.201806476.

(4) Yan, H.; Hou, Z.; Xu, H. Photoelectrochemical C-H Alkylation of Heteroarenes with Organotrifluoroborates. Angew. Chem. 2019, 131 (14), 4640-4643. https://doi.org/10.1002/ange.201814488.

(5) Perrin, D. M. [18/F]-Organotrifluoroborates as Radioprosthetic Groups for PET Imaging: From Design Principles to Preclinical Applications. Acc. Chem. Res. 2016, 49 (7), 13331343. https://doi.org/10.1021/acs.accounts.5b00398.

(6) Chansaenpak, K.; Vabre, B.; Gabbaï, F. P. [18F]-Group 13 Fluoride Derivatives as Radiotracers for Positron Emission Tomography. Chem. Soc. Rev. 2016, 45 (4), 954-971. https://doi.org/10.1039/c5cs00687b.

(7) Arduengo, A. J.; Davidson, F.; Krafczyk, R.; Marshall, W. J.; Schmutzler, R. Carbene Complexes of Pnictogen Pentafluorides and Boron Trifluoride. Monatsh. Chem. 2000, 131 (3), 251-265. https://doi.org/10.1007/s007060070101.

(8) Bolt, D. A.; Curran, D. P. 1-Butyl-3-Methylimidazol-2-Ylidene Borane: A Readily Available, Liquid N-Heterocyclic Carbene Borane Reagent. J. Org. Chem. 2017, 82 (24), 13746-13750. https://doi.org/10.1021/acs.joc.7b02730.

(9) Taniguchi, T.; Curran, D. P. Hydroboration of Arynes with N-Heterocyclic Carbene Boranes. Angew. Chem. Int. Ed. 2014, 53 (48), 13150-13154. https://doi.org/10.1002/anie.201408345.

(10) Chen, Y.; Cao, Y.; Shi, Y.; Xue, Z.; Mu, T. Quantitative Research on the Vaporization and 
Decomposition of [EMIM][Tf 2N] by Thermogravimetric Analysis-Mass Spectrometry. Ind. Eng. Chem. Res. 2012, 51 (21), 7418-7427. https://doi.org/10.1021/ie300247v.

(11) Maton, C.; De Vos, N.; Stevens, C. V. Ionic Liquid Thermal Stabilities: Decomposition Mechanisms and Analysis Tools. Chem. Soc. Rev. 2013, 42 (13), 5963-5977. https://doi.org/10.1039/c3cs60071h.

(12) Taylor, A. W.; Lovelock, K. R. J.; Jones, R. G.; Licence, P. Borane-Substituted Imidazol-2Ylidenes: Syntheses in Vacuo. Dalton Trans. 2011, 40 (7), 1463-1470. https://doi.org/10.1039/c0dt01240h.

(13) Lennox, A. J. J.; Lloyd-Jones, G. C. Preparation of Organotrifluoroborate Salts: Precipitation-Driven Equilibrium under Non-Etching Conditions. Angew. Chem. Int. Ed. 2012, 51 (37), 9385-9388. https://doi.org/10.1002/anie.201203930.

(14) Clarke, C. J.; Puttick, S.; Sanderson, T. J.; Taylor, A. W.; Bourne, R. A.; Lovelock, K. R. J.; Licence, P. Thermal Stability of Dialkylimidazolium Tetrafluoroborate and Hexafluorophosphate Ionic Liquids:: Ex Situ Bulk Heating to Complement in Situ Mass Spectrometry. Phys. Chem. Chem. Phys. 2018, 20 (24), 16786-16800. https://doi.org/10.1039/c8cp01090k.

(15) Deyko, A.; Lovelock, K. R. J.; Licence, P.; Jones, R. G. The Vapour of Imidazolium-Based Ionic Liquids: A Mass Spectrometry Study. Phys. Chem. Chem. Phys. 2011, 13, 16841-16850. https://doi.org/10.1039/c1cp21821b.

(16) Kroon, M. C.; Buijs, W.; Peters, C. J.; Witkamp, G. J. Quantum Chemical Aided Prediction of 
the Thermal Decomposition Mechanisms and Temperatures of Ionic Liquids. Thermochim. Acta 2007, 465 (1-2), 40-47. https://doi.org/10.1016/j.tca.2007.09.003.

(17) Clarke, C. J.; Bui-Le, L.; Hallett, J. P.; Licence, P. Thermally-Stable Imidazolium Dicationic Ionic Liquids with Pyridine Functional Groups. ACS Sustain. Chem. Eng. 2020, 8 (23), 87628772. https://doi.org/10.1021/acssuschemeng.0c02473.

(18) Cao, Y.; Mu, T. Comprehensive Investigation on the Thermal Stability of 66 Ionic Liquids by Thermogravimetric Analysis. Ind. Eng. Chem. Res. 2014, 53 (20), 8651-8664. https://doi.org/10.1021/ie5009597.

(19) Clough, M. T.; Geyer, K.; Hunt, P. A.; Mertes, J.; Welton, T. Thermal Decomposition of Carboxylate Ionic Liquids: Trends and Mechanisms. Phys. Chem. Chem. Phys. 2013, 15 (47), 20480. https://doi.org/10.1039/c3cp53648c.

(20) Fredlake, C. P.; Crosthwaite, J. M.; Hert, D. G.; Aki, S. N. V. K.; Brennecke, J. F. Thermophysical Properties of Imidazolium-Based Ionic Liquids. J. Chem. Eng. Data 2004, 49 (4), 954-964. https://doi.org/10.1021/je034261a.

(21) Lovelock, K. R. J.; Armstrong, J. P.; Licence, P.; Jones, R. G. Vaporisation and Thermal Decomposition of Dialkylimidazolium Halide Ion Ionic Liquids. Phys. Chem. Chem. Phys. 2014, 16, 1339-1353. https://doi.org/10.1039/c3cp52950a.

(22) Deyko, A.; Lovelock, K. R. J.; Licence, P.; Jones, R. G. The Vapour of Imidazolium-Based Ionic Liquids: A Mass Spectrometry Study. Phys. Chem. Chem. Phys. 2011, 13 (37), 16841-16850. https://doi.org/10.1039/c1cp21821b. 
For Table of Contents Use Only:

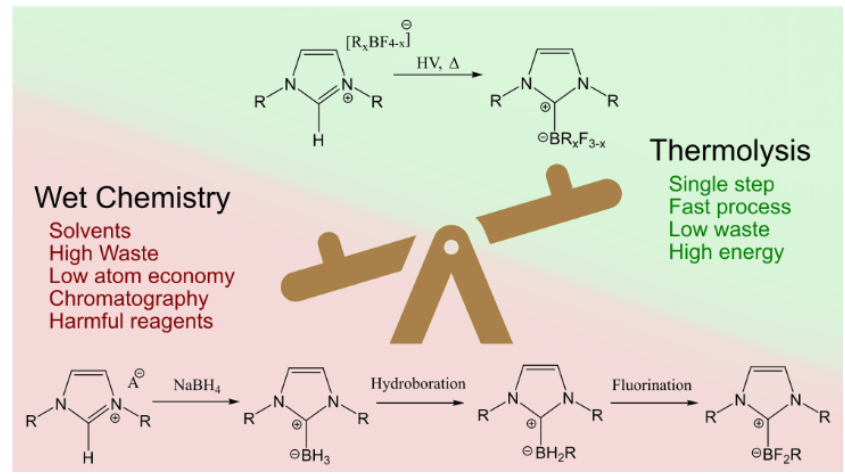

SYNOPSIS. Organofluroborates can be prepared by thermolysis of imidazolium organofluoroborate ionic liquids to avoid lengthy synthetic procedures and associated waste. 